\title{
Jets and Topography: Jet Transitions and the Impact on Transport in the Antarctic Circumpolar Current
}

\author{
ANDREW F. THOMPSON \\ California Institute of Technology, Pasadena, California, and British Antarctic Survey, Cambridge, United Kingdom \\ JEAN-BAPTISTE SALLÉE \\ British Antarctic Survey, Cambridge, United Kingdom
}

(Manuscript received 26 July 2011, in final form 13 December 2011)

\begin{abstract}
The Southern Ocean's Antarctic Circumpolar Current (ACC) naturally lends itself to interpretations using a zonally averaged framework. Yet, navigation around steep and complicated bathymetric obstacles suggests that local dynamics may be far removed from those described by zonally symmetric models. In this study, both observational and numerical results indicate that zonal asymmetries, in the form of topography, impact global flow structure and transport properties.

The conclusions are based on a suite of more than 1.5 million virtual drifter trajectories advected using a satellite altimetry-derived surface velocity field spanning 17 years. The focus is on sites of "cross front" transport as defined by movement across selected sea surface height contours that correspond to jets along most of the ACC. Cross-front exchange is localized in the lee of bathymetric features with more than $75 \%$ of crossing events occurring in regions corresponding to only $20 \%$ of the ACC's zonal extent.

These observations motivate a series of numerical experiments using a two-layer quasigeostrophic model with simple, zonally asymmetric topography, which often produces transitions in the front structure along the channel. Significantly, regimes occur where the equilibrated number of coherent jets is a function of longitude and transport barriers are not periodic. Jet reorganization is carried out by eddy flux divergences acting to both accelerate and decelerate the mean flow of the jets. Eddy kinetic energy is amplified downstream of topography due to increased baroclinicity related to topographic steering. The combination of high eddy kinetic energy and recirculation features enhances particle exchange. These results stress the complications in developing consistent circumpolar definitions of the ACC fronts.
\end{abstract}

\section{Introduction}

Mesoscale eddies play a leading role in setting the structure and magnitude of the Southern Ocean's meridional overturning circulation (MOC) (Visbeck et al. 1997; Marshall and Radko 2003; Böning et al. 2008; Hogg et al. 2008). The Southern Ocean MOC permits interactions between water masses formed at high and low latitudes and is a rate-limiting step in the ventilation of deep water as controlled by wind-driven surface convergence and divergence giving rise to subduction and upwelling (Toggweiler and Samuels 1995). Thus, inclusion of eddy fluxes in global climate models, either

Corresponding author address: Andrew Thompson, California Institute of Technology, 1200 E. California Blvd., Pasadena, CA 91125.

E-mail: andrewt@caltech.edu explicitly or as parameterizations, is necessary for accurate forecasts of future climates.

It has long been appreciated that mesoscale eddies are essential in closing the momentum budget in the Southern Ocean (Munk and Palmèn 1951; Johnson and Bryden 1989), and recent studies have emphasized that interactions between eddies and the mean flow determine the structure of the Southern Ocean MOC (Ferrari and Nikurashin 2010; Sallée et al. 2010; Naveira Garabato et al. 2011). In the Southern Ocean mesoscale eddies arise through both barotropic and baroclinic instability (Treguier et al.2007), which are sensitive to changes in the strength, orientation, and vertical structure of the mean flow. Eddy-mean flow interactions are also largely responsible for the characteristic frontal structure of the Southern Ocean's Antarctic Circumpolar Current (ACC) (Hughes and Ash 2001; Williams et al. 2007; Lenn et al. 2011; Thompson and Richards 2011). Southern Ocean 
fronts are dynamically important as they are typically associated with strong mean flows and give rise to spatial inhomogeneities in transport properties that significantly impact the large-scale meridional flux of heat, carbon, nutrients, and other tracers (Sokolov and Rintoul 2007a; Sallée et al. 2008).

The appearance of locally enhanced watermass gradients within the ACC and the implied reduction in mixing at these locations has motivated an intense effort to identify front locations, often with a goal of devising a useful coordinate system to estimate "cross front" fluxes. Early work on identifying fronts relied on definitions of property gradients as determined from hydrographic sections (Orsi et al. 1995; Belkin and Gordon 1996). These data were necessarily both temporally and spatially sparse. The introduction of satellite altimetry data enabled a more synoptic view of the fronts; the filamentary nature of these fronts and their relationship with meandering velocity jets has been highlighted (Hughes and Ash 2001; Sokolov and Rintoul 2007b). Later work by Sallée et al. (2008), Sokolov and Rintoul (2009), and Langlais et al. (2011) have indicated that the surface signature of fronts are related to subsurface hydrographic properties, which is consistent with the equivalent barotropic structure of the ACC. The strength of the fronts, as defined by the amplitude of property gradients, varies along the path of the ACC, which can be attributed to merging and splitting, meandering, or variability in forcing. Thompson (2010) has shown that part of this behavior is related to interactions of the mean flow with topographic features and the subsequent impact on nonlinear eddy-mean flow interactions.

Modulation in frontal structure along the path of the ACC is one aspect of a growing body of evidence suggesting that zonal variations in ACC characteristics are likely to play a key role in determining global properties of the Southern Ocean. Zonal asymmetry in statistical properties of the Southern Ocean was identified in early satellite altimeter data (Chelton et al. 1990), which indicated a patchy eddy kinetic energy (EKE) distribution with enhanced EKE levels typically located near western boundaries and downstream of large topographic obstacles. More recently, zonal asymmetries have been found to influence a number of Southern Ocean properties, including the response of the mixed layer to changing atmospheric wind forcing (Sallée et al. 2010), control of ACC zonal transport to wind stress curl (J. D. Zika et al. 2011, personal communication), internal potential vorticity (PV) distributions (Thompson et al. 2010), and even tidal energy dissipation and diapycnal mixing (Drijfhout and Naveira Garabato 2008). Zonal asymmetry is an important aspect to explore since most theories of the Southern Ocean overturning rate make use of the periodicity of the ACC to invoke zonally averaged theories of the MOC (Johnson and Bryden 1989; Marshall and Radko 2003; Olbers and Visbeck 2005; Nikurashin and Vallis 2011): Radko and Marshall (2006) is a notable exception.

Crucial to establishing zonal asymmetry in the Southern Ocean is the assortment of topographic obstacles that the ACC is required to circumnavigate in its path around Antarctica. Gille (1997a,b) described how local momentum balances vary along the path of the ACC, with transient eddies contributing to the maintenance of time-mean potential vorticity balances around topography. Similarly, Williams et al. (2007) proposed a stormtrack interpretation of the ACC jets and suggested that eddy-mean flow interactions provide the local acceleration or deceleration required to enable the flow to move over or around topography. Lu and Speer (2010) and Sallée et al. (2011) provide strong evidence that patterns of across-stream transport are established by topography as well. Both studies indicate that mixing is enhanced in the lee of topography.

Our goal in this study is twofold: (i) to analyze how frontal structure changes across strong transitions in topography and (ii) to determine the impact of these changes on cross-front exchange. The latter analysis involves experiments in which Lagrangian particles, or virtual drifters, are advected by velocity fields using satellite altimetry data, as well as an idealized quasigeostrophic (QG) model. The numerical model focuses on a simple bottom topography in which a wide ridge occupies a portion of a long, periodic domain. Of particular interest are regimes in which changes in bottom topography lead to a large-scale reorganization of the jet structure along the channel. This model builds on the work of Witter and Chelton (1998), who considered eddy-mean flow interactions within a QG channel with zonally varying topography. However, their domain permitted a single circumpolar jet. In the present simulations, a large domain allows for the possibility that the equilibrated number of jets is a function of longitude.

In the following section, the virtual drifter analysis is presented. Section 3 presents the results from a series of QG simulations motivated by the findings in section 2 . Particle advection experiments are also performed in the QG model. A discussion of the model and observational results is provided in section 4 , and conclusions are summarized in section 5 .

\section{Cross-frontal exchange from altimetry}

\section{a. Front detection from altimetry}

Observations from satellite altimetry have been instrumental in refining our understanding of the ACC 

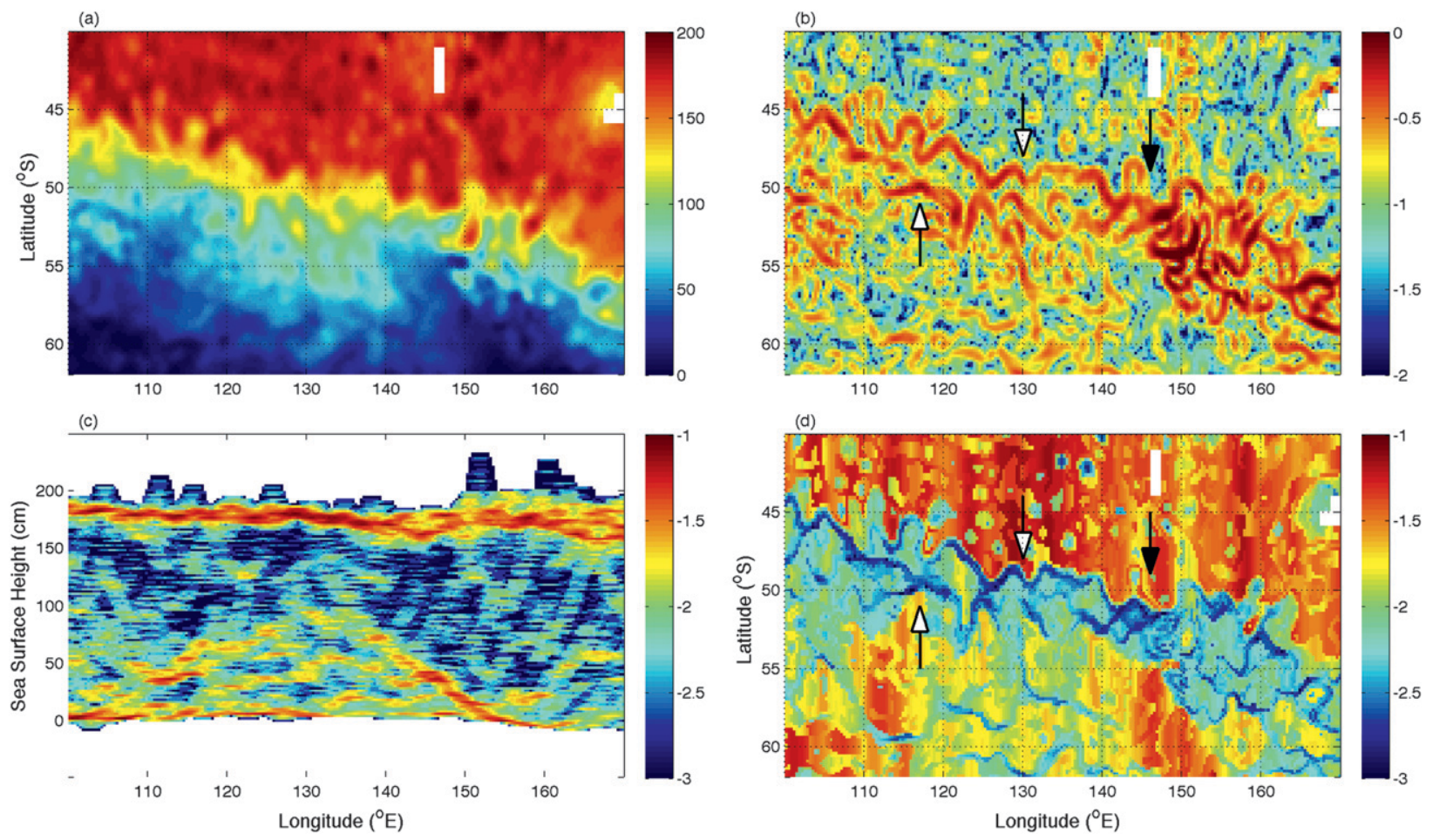

FIG. 1. (a) Snapshot of sea surface height (cm) in the Indian sector of the Antarctic Circumpolar Currnet. (b) The gradient of SSH $\left[\log _{10}(|\nabla S S H|) \mathrm{cm} \mathrm{km}^{-1}\right]$. The white arrows indicate two meandering jets, and the black arrow indicates a transition in the bathymetry at the downstream edge of the Southeast Indian Ridge. (c) Running PDF of SSH calculated from the snapshot in (a). Each vertical slice represents a PDF taken over a $3^{\circ}$ longitude window, spanning $65^{\circ}-35^{\circ} \mathrm{S}$. The PDF is normalized by the total number of data points in each window and the logarithm $\left(\log _{10}\right)$ is presented. (d) The PDF values in (c) mapped onto geographic coordinates.

frontal structure (e.g., Hughes and Ash 2001). Maps of sea surface height (SSH) and SSH gradient illustrate that the ACC comprises a sea of filamentary jets and coherent eddies. Figures 1a,b show snapshots of SSH and SSH gradient, respectively, in the Indian sector of the Southern Ocean. Between $110^{\circ}$ and $140^{\circ} \mathrm{E}$, the signal is dominated by two meandering but coherent jets, indicated by the white arrows. These features are tied to the broad Southeast Indian Ridge. The black arrow in Fig. $1 \mathrm{~b}$ indicates the location where the ridge abruptly transitions to a region of flatter bathymetry. At this point the structure of the flow changes: meanders become larger, eddies are dominant, and jets are more difficult to define.

Sokolov and Rintoul (2007b) suggest that strong gradients in SSH tend to follow specific contours of SSH. This implies that, in SSH space, certain values of SSH experience consistently stronger gradients. A challenge with using this diagnostic is that taking spatial derivatives of the altimetry field degrades the resolution of the data, which marginally resolves scales associated with Southern Ocean mesoscale. An alternative technique for highlighting regions of strong gradients is to plot the frequency at which SSH values are observed in the altimetry data or, equivalently, construct probability density functions (PDFs) of SSH. Since the ACC is partitioned into regions of strong narrow jets (high $\mathrm{SSH}$ gradient) and weaker flow (weak SSH gradients or relatively flat SSH), the former should appear as minima in the PDF calculations, whereas SSH values in weak gradient regions will appear more frequently. Figure 1c shows a "running" PDF of SSH between $65^{\circ}$ and $35^{\circ} \mathrm{S}$ constructed from the same SSH snapshot in Fig. 1a. Each vertical slice represents a PDF over a $3^{\circ}$ longitude window, normalized by the number of SSH values in the window and centered at the longitude on the abscissa (cf. Thompson et al. 2010).

While Fig. 1c does show an inhomogeneous distribution of SSH, indicative of fronts, there is little evidence of a single SSH contour that is a consistent jet over this section of the ACC. The intricacy of the ACC's front structure is captured when the PDF data is mapped back to geographic coordinates (Fig. 1d). This is done by taking the frequency values in Fig. 1c for a given longitude-SSH value and mapping it to the latitude at which the SSH contour is found at this particular time. Downstream of the obstacle (black arrow), the appearance of three or four distinct PDF minima suggest a substantial change in the front structure following the transition in 
(a)

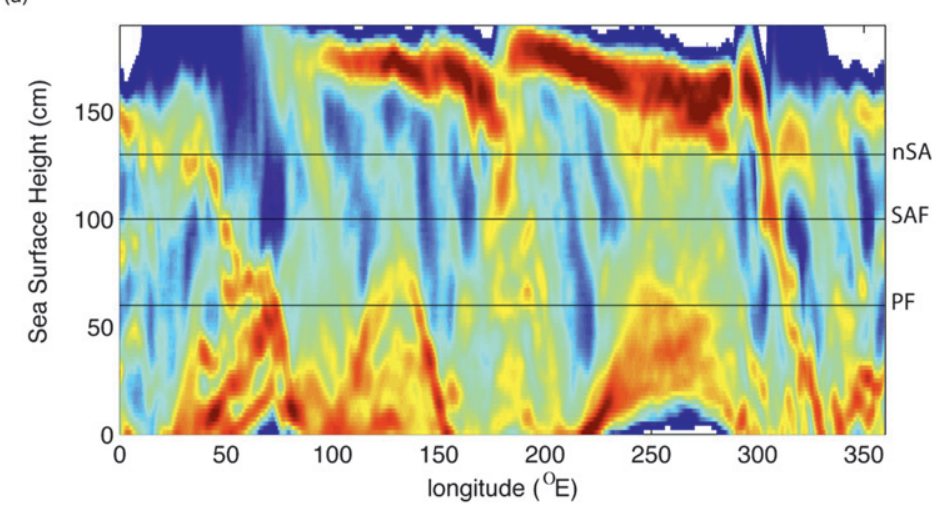

(b)
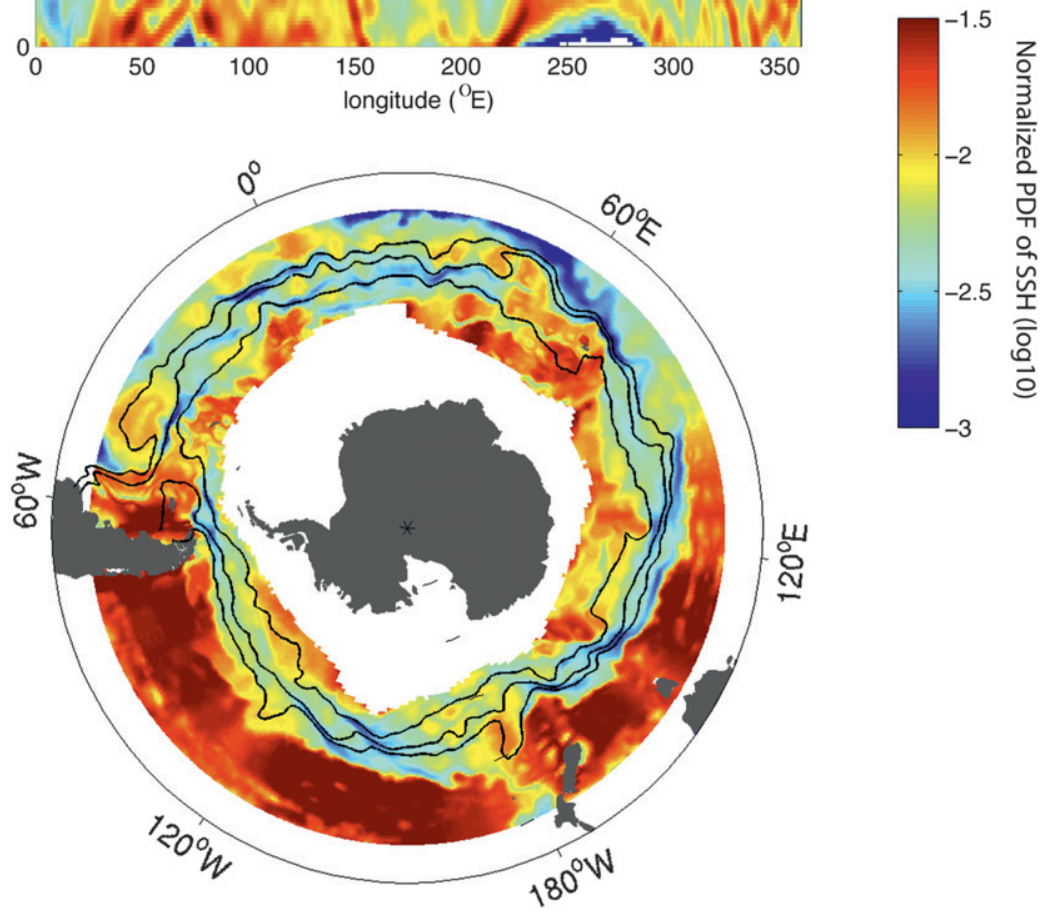

FIG. 2. (a) Running PDF of SSH (as in Fig. 1c) averaged over 17 years of satellite altimetry for the entire ACC. (b) Data from (a) mapped onto latitude and longitude coordinates by taking the mean position of each SSH value. The SSH contours associated with (from north to south) the northern Subantarctic Front, the Subantarctic Front, and Polar Front are given by the black contours.

topography. Thus, at least in this snapshot, jets are not consistently found along individual SSH contours.

This conclusion is sustained when the full $17-\mathrm{yr}$ altimetry record is used to construct a similar running PDF over the entire ACC (Fig. 2a). Conspicuous in this plot are the regions of warm colors, associated with weak SSH gradients found at large and small SSH values. These are the regions of weak flow in the midlatitude gyres to the north of the ACC and the gyres around the margins of Antarctica. There are also regions within the ACC where distinctions between maxima and minima are weak, notably near $40^{\circ} \mathrm{E}, 180^{\circ}$, and $60^{\circ} \mathrm{W}$. Once again, low values are indicative of high SSH gradients and surface velocity jets. Mapping the PDF presented in SSH space into geographic space (Fig. 2b), by using the mean position of the SSH contours, we find that changes in the distribution of SSH gradients are largely associated with major topographic features. In particular, the regions of reduced distinction between maxima and minima discussed above correspond to the Kerguelen Plateau, Campbell Plateau, and Drake Passage.

The three SSH contours plotted in Fig. 2b do a good job at tracking the position of the PDF minima in local regions of the ACC, but the plot also demonstrates that care must be taken when using circumpolar definitions of fronts. For instance, the Agulhas Retroflection, the dark blue region extending to lower SSH values between $40^{\circ}$ and $70^{\circ} \mathrm{E}$ in Fig. 2a, is an intense jet in the Southern Ocean that is not associated with a single SSH contour. While this current is not traditionally defined as one 
of the ACC fronts, it merges into the core of the ACC near the Kerguelen Plateau where many other SSH contours also merge to circumvent flowing over the plateau. Furthermore, downstream of New Zealand and Drake Passage the SSH field is distinguished by weak gradients, largely due to temporal smoothing associated with variability in the front positions over time. The SSH contours eventually return to regions consistent with fronts defined by SSH PDFs; however, it is difficult to determine whether these correspond to the same upstream fronts. Combinations of SSH and hydrographic diagnostics from either models or observations provide some support that SSH and hydrography-based definition are at least regionally consistent (Sallée et al. 2008; Langlais et al. 2011; Thompson and Richards 2011). Circumpolar definitions can be useful tools to study Southern Ocean jets; however, regional variability suggests that ACC fronts are more similar to a succession of intense jets rather than unambiguous circumpolar lines. Any cross-front transport analysis must be interpreted with this in mind.

\section{b. Particle advection scheme}

We now use the altimetry-advected virtual drifter dataset described in Sallée et al. (2011) to look at spatial variations in cross-front transport. Virtual drifters are released at each $0.1^{\circ}$ by $0.1^{\circ}$ grid point in the Southern Ocean between latitude $70^{\circ}$ and $30^{\circ} \mathrm{S}$ for a total of about 1.5 million particles over the Southern Ocean. These particles are advected using the satellite altimetry velocity field with a time step of one day and no background diffusion. Different time steps from one hour to several days have been tested for the advection scheme. The cross-front calculation converges when the advection time step reaches a value of one day, so we choose one day as the best compromise between the smallest error in the advection procedure and the best computing efficiency. Particles are advected for a period of one year, and the entire experiment is repeated every 6 months for the entire altimetry period, from 1993 to 2008. We perform a total of 32 ensemble particle releases, at the start of each winter (1 November) and summer (1 May) season.

Velocity fields are generated from 17 years of satellite altimetry data. We used weekly maps of altimetric sea level anomaly (SLA) and altimetric absolute sea surface height $\left(\mathrm{SSH}_{\mathrm{abs}}\right)$, Rio et al. (2005). The mapped SLA and $\mathrm{SSH}_{\mathrm{abs}}$ fields are provided by Collecte Localisation Satellites (CLS)/Archiving, Validation, and Interpretation of Satellite Oceanographic data (AVISO) and are based on data from the available altimeter missions [Ocean Topography Experiment (TOPEX)/Poseidon, European Remote Sensing Satellite-1 (ERS-1), ERS-2, Geosat Follow-On (GFO), Envisat, and Jason-1]. The mapping technique is described by Le Traon et al. (1998). Anomalies for SLA are calculated with respect to a 7-yr mean (1992-99) and are mapped onto a $13^{\circ}$ grid in longitude and a variable grid in latitude, ranging from approximately $1 / 20^{\circ}$ at $80^{\circ} \mathrm{S}$ to $1 / 4^{\circ}$ at $30^{\circ} \mathrm{S}$.

For flows in a chaotic advection regime, it is the largescale coarse-resolution velocity field that is the most important for tracer and particle advection (Haynes and Shuckburgh 2000; Marshall et al. 2006). This gives us confidence in advecting particles with the relatively coarseresolution altimetry data. Furthermore, Sallée et al. (2008) showed that the cross-stream diffusivity of particles advected by altimetry in the Southern Ocean is consistent with the observed diffusivity from in situ surface drifters. Despite this consistency between diffusive regimes, small errors in the advection scheme may accumulate and generate significant differences between real and simulated trajectories over six months of integration. Here, we focus on the portions of trajectories that cross fronts, which occurs in a relatively short time (a few days to a few weeks). It has been demonstrated that altimetry fields reproduce in situ trajectories well over these time scales (Nencioli et al. 2011).

Sallée et al. (2011) used the virtual drifter dataset to compute eddy-induced diffusion along particle trajectories. They describe a complex lateral mixing distribution resulting from a competition between mean-flow mixing reduction and a bathymetrically induced increase of mixing. Here we analyze the distribution of cross-front exchange by identifying the sites where particles cross individual fronts. This method produces a complementary but unique picture by providing a much finer spatial resolution of transport characteristics, as it avoids the necessity of integrating along trajectories associated with the diffusivity calculations. To remain consistent with previous work on Southern Ocean front structure, we define three fronts to monitor: the northern branch of the Subantarctic Front (nSAF), the Subantarctic Front (SAF), and the Polar Front (PF). Fronts are defined using SSH contours following Sallée et al. (2008).

\section{c. Altimetry results}

Figure 3 provides an estimate of the number of particles that cross the three primary fronts of the ACC: the nSAF (top), the SAF (middle), and the PF (bottom). The detection algorithm selects only those particles that cross a given SSH contour and change their associated $\mathrm{SSH}$ value by more than $5 \mathrm{~cm}$ (black curve), $10 \mathrm{~cm}$ (red curve), or $15 \mathrm{~cm}$ (blue curve). These different exchange criteria modulate the number of particles deemed to have crossed a front, but the spatial pattern of the exchange is insensitive to this value. Particle exchange is 
(a)

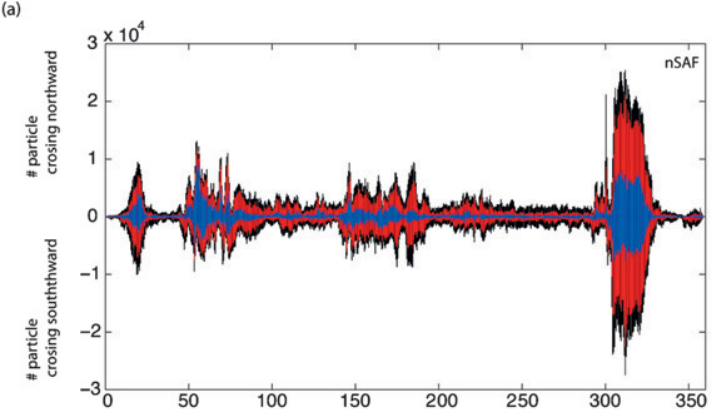

(b)

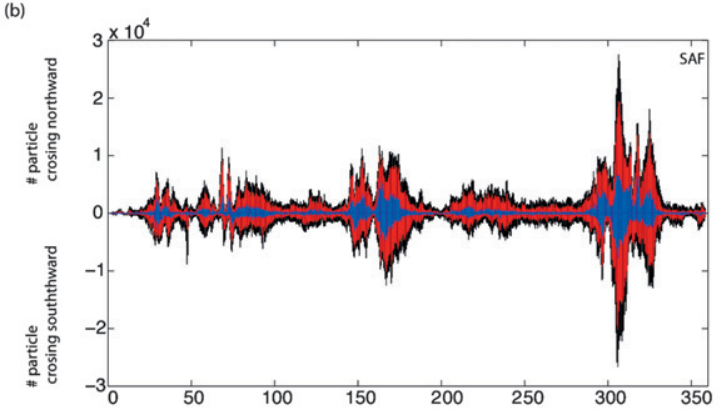

(c)

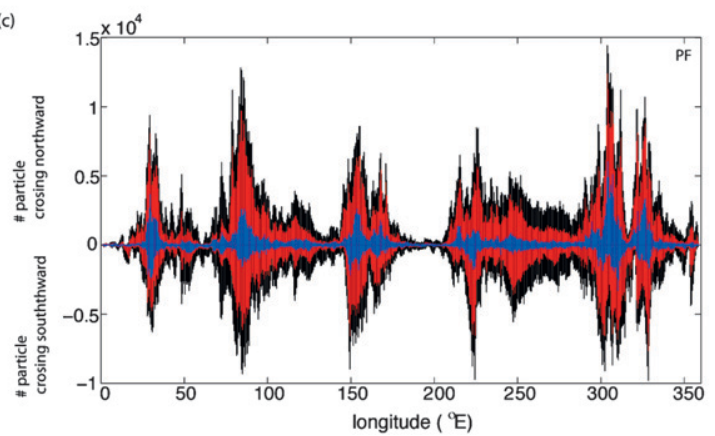

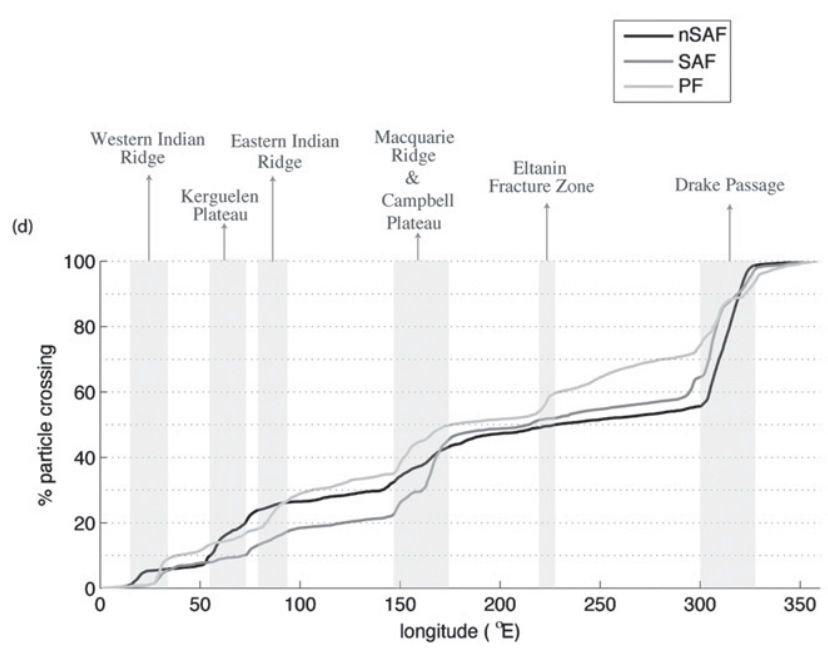

(e)

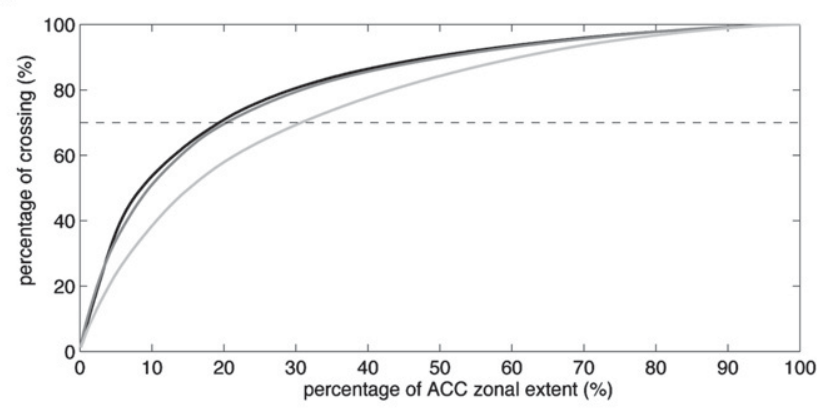

FIG. 3. Number of Lagrangian particles that cross the (a) nSAF, (b) SAF, and (c) the PF. Particles are determined to have crossed the front if they change their SSH value by 5 (black), 10 (red), or 15 (blue) $\mathrm{cm}$. (d) Cumulative percentage of particle crossings as a function of longitude. Shaded bars indicate regions with major topographic obstacles. (e) Cumulative percentage of particle crossing as a function of zonal extent of the ACC.

characterized by spatial localization, manifested by large spikes separated by regions of relatively weak exchange. In general, particle exchange increases abruptly on the upstream side of large exchange sites and decays more slowly downstream. This is especially true of particles crossing the SAF and PF in the Indian and Pacific sectors of the Southern Ocean. We note that there are roughly the same number of particles moving northward and southward across the fronts, leading to a small residual net exchange.

The discretization of the particle exchange along the path of the ACC suggests that local dynamics contribute substantially to global transport properties. A crude way of estimating this contribution is to regard each particle crossing as an "event" and then to integrate the number of events occurring within different regions of the ACC. Figure $3 \mathrm{~d}$ shows the cumulative distribution of particle crossing events for the three fronts. There is good correlation between regions of particle exchange and topographic features (shaded regions). The largest contribution for all fronts comes from Drake Passage, although some meridional variations occur: particles cross the nSAF in one wide region spanning $60^{\circ}-30^{\circ} \mathrm{W}$, whereas transport across the SAF and the PF occurs in a series of discrete bursts. The narrow minima in $\mathrm{PF}$ crossing events at $315^{\circ} \mathrm{W}$ (Fig. 3c) is correlated with the front crossing the Scotia Arc. In each case roughly $70 \%$ of the crossing events, indicated by the dashed line in Fig. 3e, occur over only $20 \%$ of the zonal extent of the ACC: $19 \%, 20 \%$, and $31 \%$ for the SAFN, SAF, and PF; $75 \%$ of crossing events over $24 \% ; 25 \%$ and $37 \%$ of the zonal extent for the same fronts.

Figure 4 presents further information about the particle exchange by mapping the crossing events onto the 


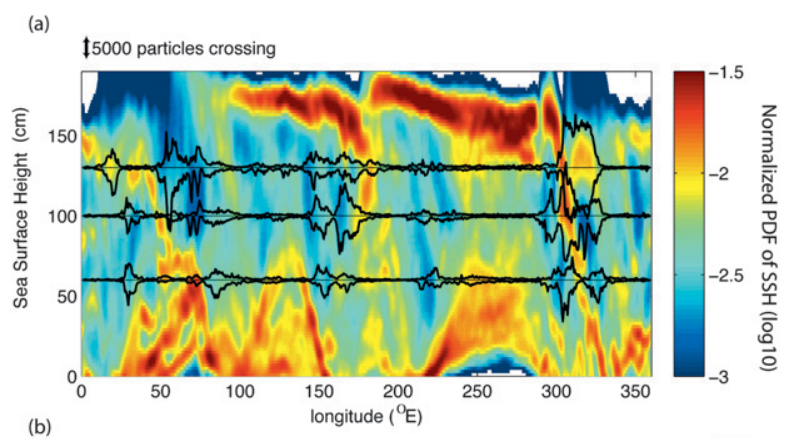

(b)
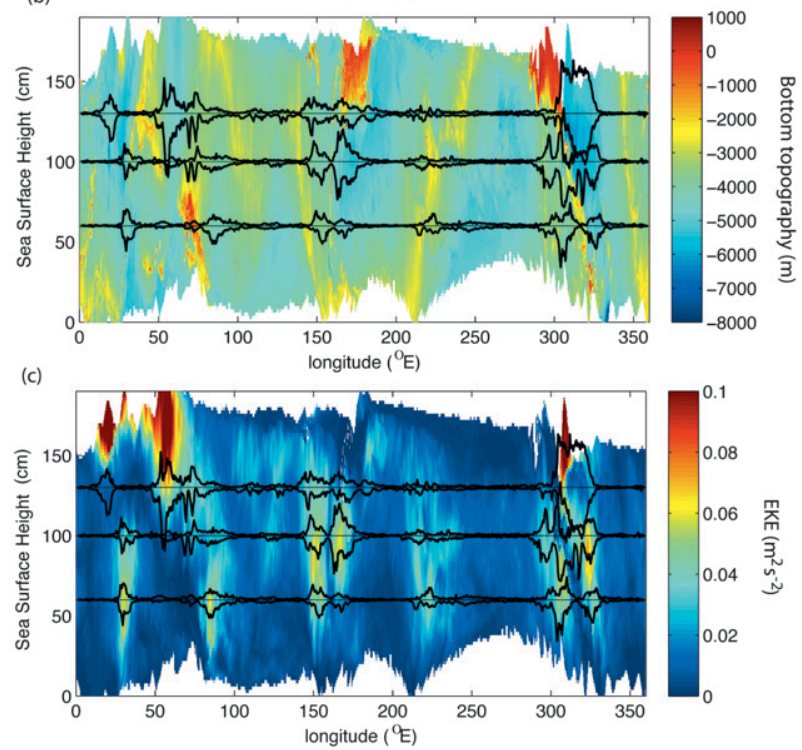

FIG. 4. The number of particles crossing, from top to bottom, the nSAF, SAF, and PF mapped over (a) the time-averaged PDF of $\mathrm{SSH},(\mathrm{b})$ Southern Ocean bathymetry (m), and (c) EKE $\left(\mathrm{cm}^{2} \mathrm{~s}^{-2}\right)$. The upper and lower curves represent particles moving from south to north and from north to south, respectively.

time-averaged PDF of SSH (Fig. 4a), the bathymetry (Fig. 4b), and EKE (Fig. 4c). In each case, the quantities are presented in SSH space rather than geographic coordinates. As emphasized earlier, the SSH contours selected to represent the front positions in this study do not correspond to uniformly low values in the SSH PDFs (Fig. 4a) or, equivalently, are not consistently associated with strong SSH gradients (Sallée et al. 2008). Particle exchange is in general enhanced both upstream and downstream of zonal transitions in the PDF, which is indicative of a reorganization of the front structure.

Correlations are also apparent between particle exchange and bathymetric features or EKE. Mapping bathymetry into SSH space shows an enhancement in particle crossing downstream of topographical features. Although this enhancement occurs at the more prominent features along the ACC, such as Kerguelen Island and Drake Passage, smaller features such as the Mid-Atlantic
Ridge, the Eastern Indian Ridge, and the Mid-Pacific Ridge also appear to generate substantial crossing of particles. This picture is consistent with the idealized study of MacCready and Rhines (2001), who showed that meridional transport is enhanced immediately downstream of topographic features due to both a bolus flux and lateral Reynolds stresses. Finally, while the particle crossing largely occurs downstream of topography, regions of high particle exchange also correlate with sites of enhanced EKE. It is well known that EKE is enhanced downstream of topographical features, but this analysis provides further evidence that these sites are likely to play a key role in the global transport of watermass properties across the ACC's major fronts.

Advection of particles using the altimetric velocity fields provides strong observational evidence that crossfront Lagrangian particle exchange-and likely crossfront watermass transport if we assume an equivalent barotropic structure-experiences spatial variations along the path of the ACC. Questions remain as to why particle exchange occurs at these sites. Among the possible hypotheses is that the exchange is simply related to enhancement of EKE in the lee of topographic features. Alternatively, the effectiveness of the fronts as barriers to transport may weaken here (Ferrari and Nikurashin 2010), or potentially the frontal structure undergoes a more fundamental change. These questions motivate the introduction of an idealized QG model in the following section, with a specific focus on the evolution of front structure across transitions in bottom topography. The results point to the importance of front transitions in enabling particle exchange.

\section{Jet transitions in an idealized model}

We now discuss a series of numerical simulations designed to address issues of transitions in jet structure. We consider a two-layer QG model since it is the simplest model that captures the dynamics related to front transitions near topography. Limitations of the QG model are discussed in more detail in section 4. Still, QG has historically proven to be an important test bed for understanding how coherent structures determine the structure and statistical properties of turbulent geophysical flows. In particular, this study builds on modeling work by Witter and Chelton (1998) and Bracco and Pedlosky (2003) in which topography impacts the flow through the modification of potential vorticity gradients that feed back on linear instability and nonlinear turbulent properties of the flow. This paper focuses on simulations where topography catalyzes a reorganization of the front structure. 


\section{a. Model configuration}

We consider the two-layer QG model on a $\beta$ plane. The domain is doubly periodic to allow for multiple jets to form without the added complications of interactions with boundaries to the north and south (Pavan and Held 1996). The main experiments that we discuss here use a domain that is four times longer in longitude than in latitude. This greater zonal extent enables the jets to equilibrate downstream of topographical transition regions. For simplicity we assume equal layer depths $H_{1}=H_{2}=H$. Potential vorticity $q$ is advected by a two-dimensional, incompressible flow in each layer $\left[u_{i}=-\psi_{i y}, v_{i}=\psi_{i x}\right.$, where $\psi_{i}$ is the upper- $(i=1)$ and lower- $(i=2)$ layer streamfunctions] and experiences dissipation at small and large scales;

$$
\begin{aligned}
q_{i} & =\nabla^{2} \psi_{i}+\frac{(-1)^{i}}{2 \lambda}\left(\psi_{1}-\psi_{2}\right)+f+\delta_{2 i} \frac{f_{0}}{H} \eta, \\
\frac{D q_{i}}{D t} & =-\kappa \delta_{2 i} \nabla^{2} \psi_{2}+\mathrm{ssd}, \\
\frac{D}{D t} & \equiv \frac{\partial}{\partial t}+\psi_{x} \frac{\partial}{\partial y}-\psi_{y} \frac{\partial}{\partial x} .
\end{aligned}
$$

Here $\lambda$ is the baroclinic deformation radius, $\kappa$ is the linear bottom friction coefficient, $\delta_{i j}$ is the Kroenecker delta, $f$ is the Coriolis frequency, and ssd indicates smallscale dissipation implemented with a high-wavenumber filter (Smith et al. 2002). For further details on the model and assumptions, see Pedlosky (1987) and also Thompson (2010) for a specific case with bottom topography.

Background gradients in PV arise from three contributions. The first is the large-scale planetary PV gradient, represented by the linear contribution to the Coriolis frequency $f=f_{0}+\beta y$. The second contribution comes from the imposed vertical shear, $\left\langle\psi_{1 y}-\psi_{2 y}\right\rangle=$ $-2 U$, where angle brackets indicate a spatial average over the domain. This shear provides the source of available potential energy (APE) that is converted to kinetic energy through baroclinic instability. The final contribution comes from the topography, $\eta(x, y)$, which has a zonal $\mathcal{Z}(x)$ and a meridional $\mathcal{M}(y)$ component:

$$
\begin{gathered}
\eta(x, y)=\frac{s L_{y}}{2 \pi} \mathcal{Z}(x) \mathcal{M}(y), \\
\mathcal{M}(y)=\left[1-\cos \left(2 \pi y / L_{y}\right)\right],
\end{gathered}
$$

Here $L_{x}$ and $L_{y}$ are the zonal and meridional domain extents, $\gamma$ determines the zonal extent of the ridge, $\sigma$ determines the extent of the transition region between the ridge and the flat bottom, and $s$ determines the steepness of the slope. For the studies discussed here, $L_{x}=256 \lambda, L_{y}=64 \lambda$, and $\sigma=5 \lambda$. Along with full ridge and flat bottom simulations, $\gamma=3 L_{x} / 8, L_{x} / 4$, and $L_{x} / 8$ corresponds to simulations with a ridge extending $1 / 4,1 / 2$, and $3 / 4$ of the domain. The equations are nondimensionalized using $U$ and $\lambda$; we set $\kappa \lambda / U=0.1$ and $\beta \lambda^{2} / U=$ 0.75 . The main experiments have a resolution of $1024 \times$ 256; simulations have been conducted at double this resolution, which does not change the results reported here.

\section{b. Front structure}

Thompson (2010) showed that, when topographic features have a meridional extent comparable to the jet separation, the jets exhibit strong temporal variability. Here, the meridional extent of the ridge is much greater than the jet spacing such that the jet positions are steady and localized by the topography. Alongstream transitions in the jet structure occur, however, as the flow adjusts between the ridge sector, where topography enhances and opposes $\beta$ on opposite flanks, and the flat bottom sector. In the following section, we focus our analysis primarily on two simulations, one with a significant reorganization of the jets (experiment A: $\gamma=$ $L_{x} / 4$ and $s=3$ ) and a second with predominantly periodic jets (experiment B: $\gamma=3 L_{x} / 8$ and $s=3$ ). Experiment A is representative of other simulations in which front transitions were observed.

Figure 5a shows a snapshot of upper-layer PV from experiment A, illustrating the development of homogenized potential vorticity regions separated by strong gradients. The position, spacing, and strength of these mixing barriers are strongly influenced by topography. In particular, on the northern flank of the ridge the partitioning of PV is seen more clearly at the downstream edge of the ridge $(x / \lambda \approx 50)$ rather than at the upstream edge. Thompson et al. (2010), using output from a highresolution ocean GCM, showed that the position of fronts can be accurately tracked by calculating PDFs of potential vorticity (cf. Fig. 1). We use the same technique here to identify front positions in the QG numerical model. A PDF of PV values is first calculated over a window 

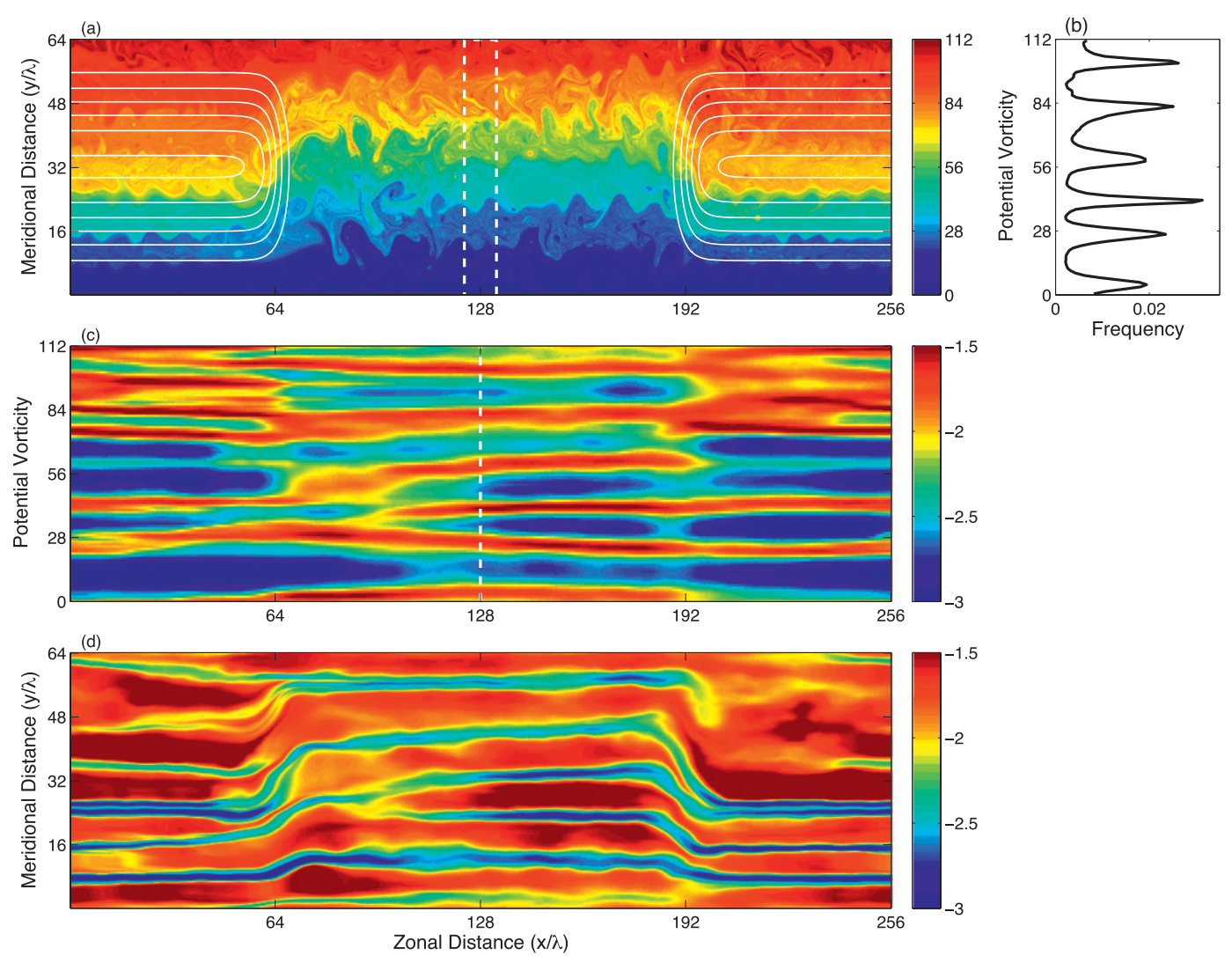

FIG. 5. (a) Snapshot of the upper-layer PV $\left(q_{1} \lambda / U\right)$ from a simulation with a zonal ridge that occupies half of the domain. The thin lines indicate the ridge topography. (b) The PDF of PV values corresponding to the window indicated by the dashed lines in (a). (c) The accumulation of the PV PDFs from (a) as the window is shifted zonally. Each slice represents a calculation over a window with a $10 \lambda$ zonal extent; the color scale is logarithmic. The PDF in (b) is mapped to the dashed line in (c). (d) PDF values as in (c) but mapped back to the mean latitude of each PV contour. Minima are indicative of jet cores or transport barriers.

with a zonal extent of $10 \lambda$ (dashed lines). Figure $5 \mathrm{~b}$ shows an example PDF for the window in Fig. 5a; the PDF is normalized by the number of grid points so that the value reported is the frequency with which a given PV value appears in a window. The window is then shifted over the full zonal extent to create a running PDF of PV values as shown in Fig. 5c. The dashed line in Fig. $5 c$ corresponds to the PDF in Fig. 5b.

Replacing SSH gradients and SSH contours with PV gradients and PV contours, it is again the case that fronts do not necessarily coincide with particular PV contours throughout the entire domain (Sokolov and Rintoul 2007b, 2009). This implies that fronts or possible transport barriers must have limited zonal extents. This is further emphasized when Fig. 5c is mapped back onto the mean latitude of each of the PV contours (Fig. 5d); the fronts or transport barriers are indicated by local minima. The barriers are enhanced on the southern flank of the jet, but downstream of the topography they are displaced northward due to conservation of PV. The combination of this jet displacement and the modified background PV gradient leads to a reorganization of the jet positions as well as the formation of new barriers. Thus, there are a different number of coherent jets over the ridge and over the flat-bottom regions. Only the southernmost front, which occurs near the latitude where topography varies weakly, is a true circumpolar feature.

The front structure depends crucially on the amplitude and zonal extent of the topographic ridge. Figure 6 shows similar PDF calculations for a series of simulations in which the amplitude of the topography is held fixed $(s=3)$ but the zonal extent of the ridge is varied. Although experiment A (Fig. 6c) is the most extreme case in terms of jet reorganization, all simulations show some evidence of downstream evolution when topography is zonally asymmetric. The importance of these transitions on particle transport is the focus of the next section.

Reorganization of the jet structure is concentrated in topographic transition regions. To examine the dynamics 

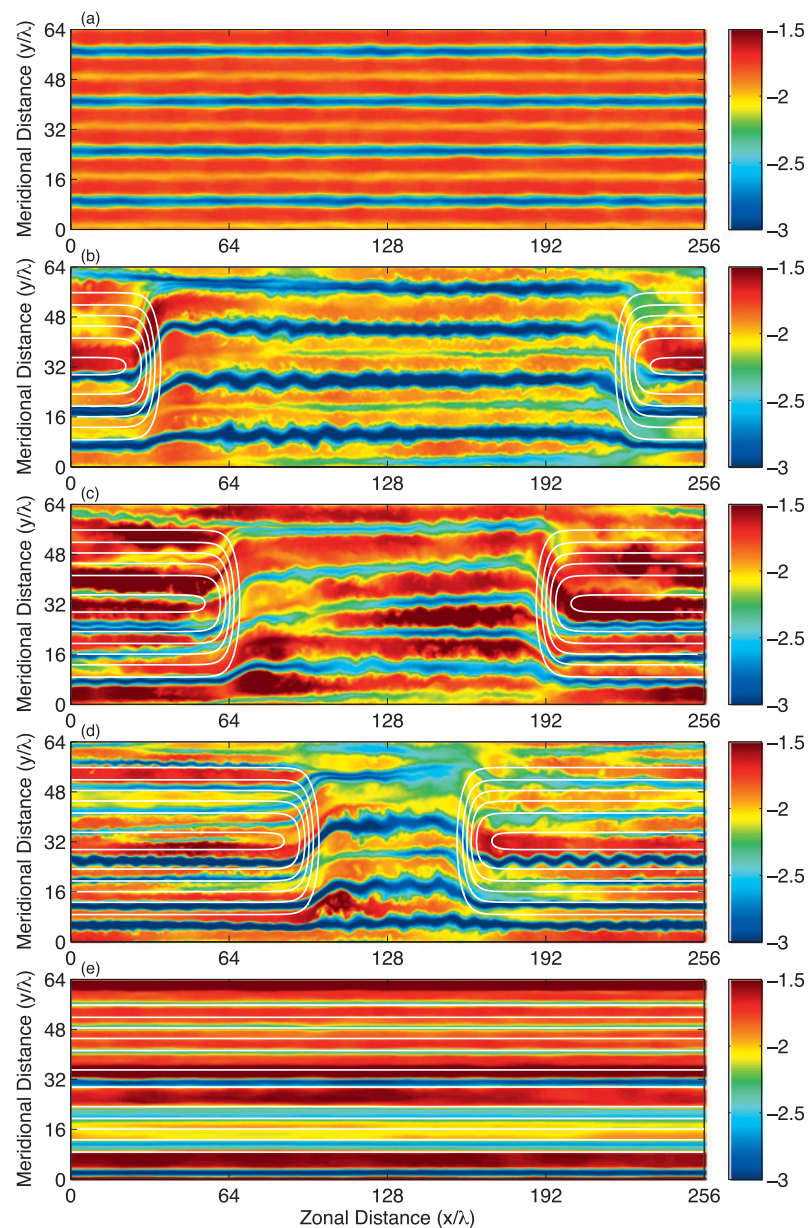

FIG. 6. Comparison of running PDFs of the upper-layer PV $\left(q_{1} \lambda / U\right)$ mapped to the mean latitudes of PV contours (as in Fig. 5d). The ridge steepness is held fixed, $s=3$, and the extent of the ridge varies: (a) flat bottom, (b) quarter domain, (c) half domain, (d) threequarter domain, and (e) full domain. The white contours indicate the bathymetry; the color scale is logarithmic $\left(\log _{10}\right)$.

of these transitions, we consider eddy forcing of the mean flow in Fig. 7. In Fig. 7a, the color represents the EKE of the barotropic mode. Large EKE values are concentrated in the core of the jets, largely because of fluctuations in the strong zonal flows. Maxima in EKE, however, are concentrated in two regions immediately downstream of the ridge and are associated with a meridional shift of the mean flow after it transitions between the ridge and the flat bottom topography. In both cases, the elevated EKE levels follow immediately downstream of sites with large barotropic mean kinetic energy (not shown), indicating an exchange between the mean and eddy components at this location. To focus on the spatial structure of this exchange, we highlight the regions surrounding three jets found along $q_{1} \lambda / U=16,52$, and 68 and calculate Reynolds stresses in these regions.
The white bold lines in Fig. 7a denote regions within \pm 6 $U / \lambda$ units of these jet positions.

Upper-layer Reynolds stresses are first calculated by determining eddy velocities as a departure from a time mean, $\mathbf{u}^{\prime}(\mathbf{x}, t)=\mathbf{u}(\mathbf{x}, t)-\overline{\mathbf{u}}^{t}(\mathbf{x})$, using 400 snapshots in the statistically equilibrated state. The eddy velocities are then locally rotated into components along $\left(u^{*}\right)$ and across $\left(v^{*}\right)$ mean PV contours. ${ }^{1}$ The color in Fig. $7 \mathrm{~b}$ then shows the gradient of the cross-frontal flux of alongfront eddy momentum,

$$
\mathcal{R} \equiv-\left({\overline{u^{*} v^{*}}}^{t}\right)_{y} .
$$

Here $y$ is taken to be in the direction normal to the PV contours. A further zonal average is carried out over an extent of $50 \lambda$ along mean PV contours before plotting in Fig. 7b.

There are distinct transitions in the eddy forcing of the mean flow. Most notably, the mean flow is decelerated at the downstream transition in topography. Topographic steering plays a key role in the exchange of energy between eddies and mean flow in this region. Similar to Boland et al. (2012), the lower layer is steered more strongly by the ridge at the downstream edge, leading to a tilting of the mean flow orientation with depth and an increase in baroclinicity of the mean flow. In this nonzonal configuration the flow is efficient at releasing APE (Arbic and Flierl 2004; Smith 2007), leading to enhanced eddy generation in the lee of topography. These same eddies are then advected downstream and transfer their energy back into the mean flow throughout most of the flat bottom sector of the domain. Eddy forcing at the upstream transition region also helps to accelerate the jets onto the ridge (Williams et al. 2007), where Reynolds stresses are considerably weaker. This figure clearly illustrates the storm track interpretation of jet dynamics described in Williams et al. (2007) as eddies act to both accelerate and decelerate the mean flow in different regions.

\section{c. Particle tracking}

We generate Lagrangian particle trajectories in two experiments, A (Fig. 6c) and B (Fig. 6b), for the purpose of comparison with the observational results. These experiments have different characteristics. In experiment $\mathrm{A}$, there is a clear reorganization of the jets at the transition regions, with the number of jets varying with longitude. On the other hand, jets in experiment B

\footnotetext{
${ }^{1}$ The calculation was also carried out using mean streamlines. The results are qualitatively similar but less clear in the case with streamlines due to recirculation features downstream of topography that lead to closed streamlines.
} 

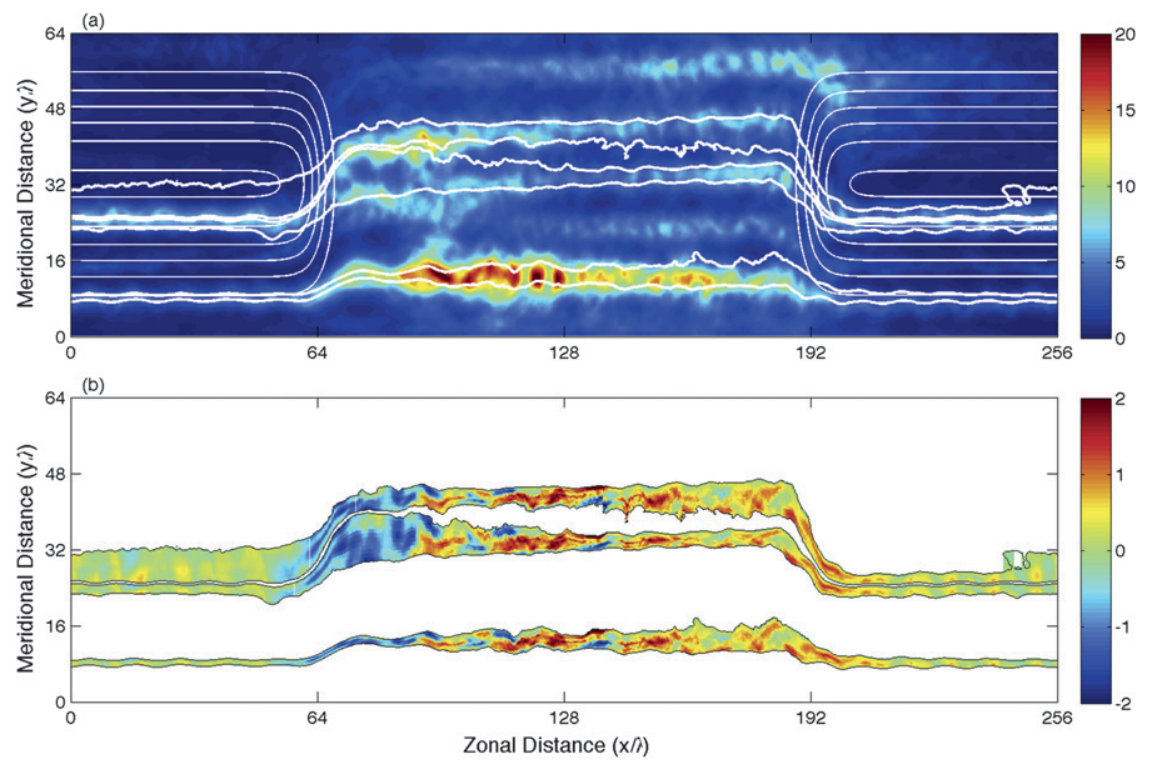

FIG. 7. (a) Time-averaged barotropic EKE from experiment A; energy is nondimensionalized by $U^{2}$. The thin white contours indicate the topography; the bold white contours represent regions $\pm 6 U / \lambda$ PV units away from jets along $q_{1} \lambda / U=16,52$, and 68. (b) The spatial distribution of the time-averaged Reynolds stresses $\mathcal{R} \lambda / U^{2}[(7)]$ from the same experiment. The contoured regions correspond to the bold white contours in (a).

undergo significant deflection downstream of topography but remain largely "circumpolar," or periodic. During the statistically equilibrated state, 500000 particles are released and advected for a period of $20 \lambda / U$. At the end of this period, new particles are seeded and the experiment is repeated six times for a total of three million particle trajectories. The advection of the particles is carried out in the upper layer and PV is tracked along the trajectories.

Figure 8a shows the PDF of upper-layer potential vorticity in experiment $A$; its zonal average is shown in Fig. 8b. PDF minima in Fig. 8b and their corresponding PV values are taken to represent global transport barriers, similar to identifying SSH with fronts in section 2 (results are qualitatively similar if streamfunction contours are used as front definitions). In experiment $\mathrm{A}$, the minima do not always correspond with circumpolar fronts. In Figs. 8c-j, we show the number of particles crossing a given PV contour moving both southward and northward. Along most of the PV contours, there are significant zonal variations in the particle crossing, similar to the results from the altimetry data. In particular there are enhanced crossing events just downstream of the ridge across barriers $d$ and e. Transitions in exchange characteristics between the ridge and the flat-bottomed sectors are apparent across lines $\mathrm{d}$ through $\mathrm{g}$.

In Fig. 9, the zonally averaged minima of the PDF of PV from experiment B (Fig. 9b) are more pronounced, which is reflected in the smaller number of particles crossing the domain. Still, there are marked spatial transitions with very few particles crossing on the southern flank of the ridge and most of the transfer occurring within the flat-bottomed region. The largest transition occurs along the northern flank of the ridge, where the transport barrier effectively disappears.

The results from Figs. 8 and 9 show that there is a strong correlation between particle crossing and the amplitude of the PV PDFs. In particular, in experiment A, the largest transfer of particles along $q_{1} \lambda / U=52$ occurs on the downstream edge of the ridge, where the PDF has its maximum value corresponding to the region of jet reorganization. Particle exchange also correlates with transitions in the bathymetry, with sharp increases in particle crossing at the ridge's downstream edge over most of the domain. Surprisingly, there is only weak correlation between the EKE and the particle crossing; this is discussed further in section 4 . In experiment $\mathrm{B}$, the strong correlation of particle exchange with the PDF and topography is also observed. Particle crossing is roughly uniform throughout the flat-bottom region here, whereas the EKE levels decay throughout the flatbottom sector.

\section{Discussion}

\section{a. Comparison between observations and model}

In the previous sections, we have presented results from two very different datasets: one based on satellite 

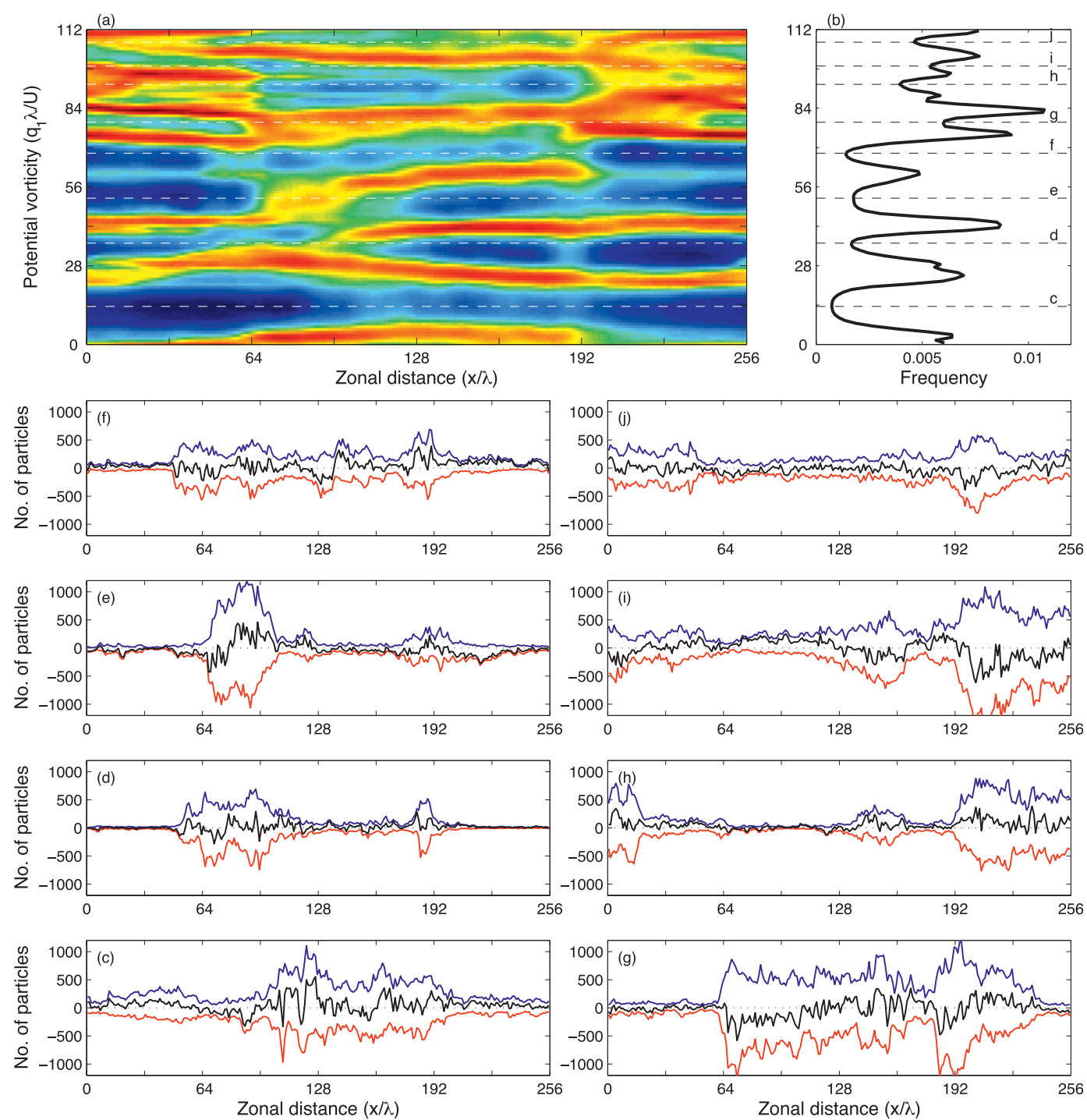

FIG. 8. (a) Running PDF of the upper-layer PV $\left(q_{1} \lambda / U\right)$ for experiment A (as in Fig. 6c). The white dashed lines indicate locations of PDF minima following an along-PV mean (b). (c)-(j) The number of particles crossing the associated PV minima, or transport barrier, indicated in (b). The blue, red, and black curves indicate particles moving northward and southward and their sum, respectively.

altimetry data providing realistic surface velocities from the Southern Ocean and a second based on an idealized model with simple topography (that conforms to QG scalings) and a vertical truncation to two layers. Thus, a few words are required as to how these two studies relate to each other.

First, it is important to acknowledge that the QG model is not a faithful representation of the Southern Ocean. In particular, the model is derived assuming that modulations in topography are small compared to layer depth, which is certainly not true in the ACC. Furthermore, the parameters $\beta \lambda^{2} / U$ and $\kappa \lambda / U$ are chosen to produce a simulation with multiple jets in the case with flat bottom topography. In general, $\beta \lambda^{2} / U$ is much weaker in the Southern Ocean $(\sim 0.1)$, but QG simulations at these values do not result in multiple coherent jets (Thompson and Young 2007). This discrepancy may be explained by the fact that topographic $\beta$ often makes the dominant contribution to the large-scale PV gradients in the Southern Ocean. Finally, equal layer depths are largely chosen for convenience, as we are focusing on an idealized setting. A more realistic stratification would be worth exploring since it will affect communication between the model layers and topography. However, changing layer depths in similar QG models tends to produce qualitatively similar results (Arbic and Flierl 2004). 

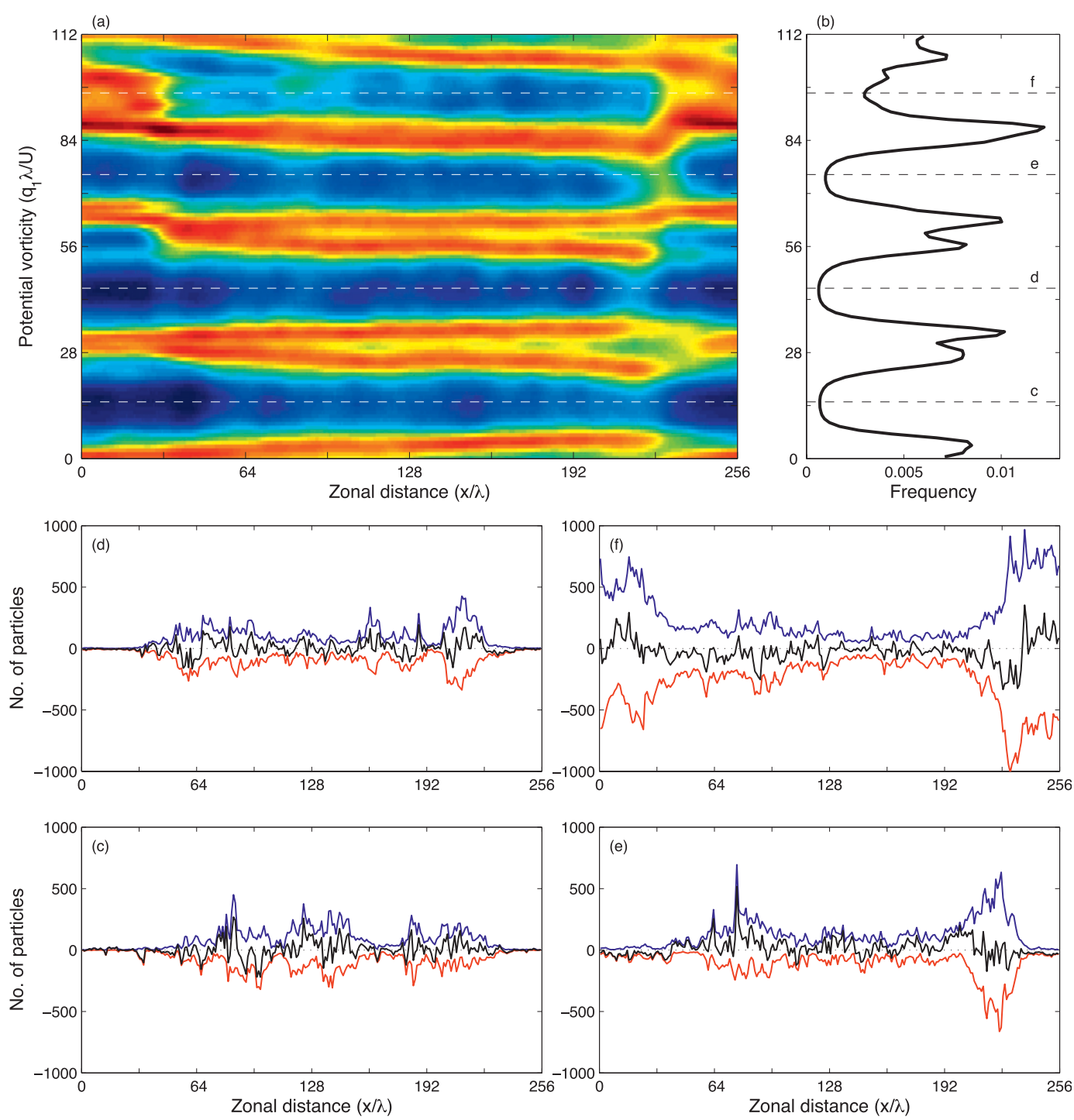

FIG. 9. As in Fig. 8 but for expt B (Fig. 6b) with a zonal ridge that occupies one quarter of the domain. The color scale for (a) is as in Fig. 5. Jets here have a strong circumpolar character.

The advantage of using the QG model is that it allows a process-oriented approach, where the relationship between eddy-mean flow interactions and topography can be investigated. QG dynamics are sufficiently complex to represent the barotropic and baroclinic instability processes responsible for much of the eddy activity in the Southern Ocean. Thus, the results from the QG model are complementary to the observational results, but future simulations should address the vertical structure of the transport properties in the presence of more realistic topography.

In the statistically equilibrated state of the QG simulations, the downstream edge of the ridge is a site of enhanced EKE. As the jet moves off the ridge, it deflects northward in order to conserve PV; the extent of the deflection increases for jets located higher up the ridge (Fig. 5d). This deflection causes the jets on the southern flank of the ridge to diverge, which leads to the generation of recirculation features immediately in the lee of the ridge in experiment A. Regions of multiple closed streamlines are found predominantly in the downstream transition zone. The barotropic streamfunction from experiment $B$ has weaker recirculation features with closed streamlines largely confined to standing meanders of the jets. The combination of enhanced EKE and the formation of recirculation features are also key ingredients determining mixing and transport properties of the Southern Ocean. While EKE levels are observed to be elevated in the lee of topography (Chelton et al. 2007), Sallée et al. (2011) show that 
(a)
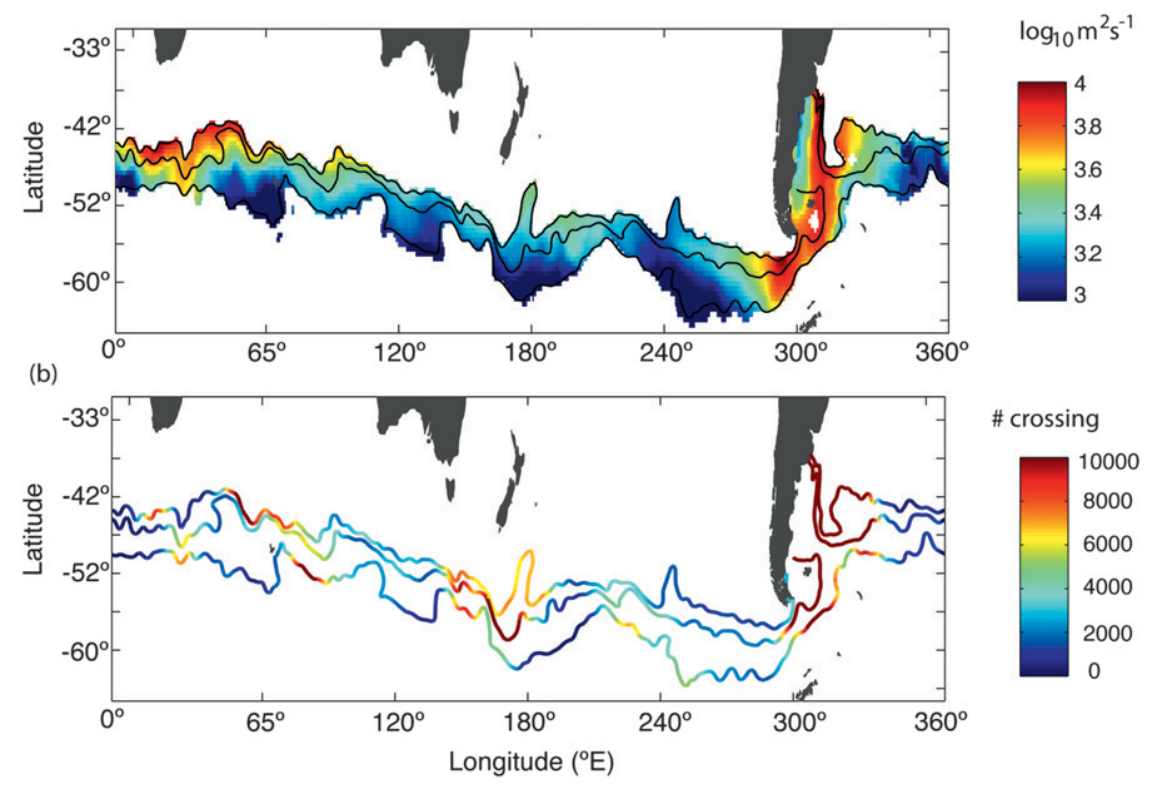

FIG. 10. (a) Eddy diffusivity within the ACC calculated from integrating along Lagrangian particle trajectories (Sallée et al. 2011). (b) Number of particle crossings across (from north to south) the nSAF, SAF, and PF, as a function of longitude based on the same particle trajectories.

enhanced EKE levels are not sufficient by itself to imply large eddy diffusivities or, indeed, large particle exchange. Instead, large EKE levels must also coincide with "eddy stagnation" regions, which correspond to sites where the advection of anomalies in the Southern Ocean are weak (Fu 2009).

Figure 10 provides a direct comparison between the particle exchange calculated in this study and the eddy diffusivities in Sallée et al. (2011). In general, there is good agreement between the two, although regions exist where the eddy diffusivity and the front crossings differ. The advantage of the particle exchange calculation is that it is more effective at identifying regional processes. For instance, the Agulhas Retroflection affects eddy diffusivity values south of South Africa within the fronts (the red in Fig. 10a) between $20^{\circ}$ and $60^{\circ} \mathrm{E}$. However, this may be an artifact of the smoothing that occurs owing to integration along trajectories. Indeed, the cross-front calculation shows that there are only isolated regions of cross-front exchange (at $25^{\circ} \mathrm{E}$, at the Western Indian Ridge, in agreement with eddy diffusivity). Eddy diffusivities are smoother, whereas the cross-front results exhibit more abrupt transitions. We believe that these two approaches are complementary: one provides a flux estimate but blurs regional structure due to integration over long trajectories, whereas the second provides only qualitative information about the flux but provides a more sharply resolved picture of regional variability.
The largest discrepancy between the model and observational results is the relationship between EKE and particle crossing. In the altimetry experiments, the sites of particle exchange are strongly correlated with high levels of EKE (Fig. 4c). In experiment A, the largest EKE is found along the $q_{1} \lambda / U=16$ contour (Fig. 7a), but here the jet remains a moderately strong barrier to transport (Fig. 8c). A second EKE maximum along the $q_{1} \lambda / U=60$ contour is weaker, but it is found within a recirculation feature that aids the localized particle exchange across the jet at $q_{1} \lambda / U=60$ (Fig. 7e). Existing theories about eddy diffusivities in the ACC rely primarily on traditional mixing length arguments that require a scale separation between eddies and mean flow. Ferrari and Nikurashin (2010) include the effects of the mean flow in their mixing length theory, showing that the mean flow can suppress mixing even in high EKE regions. This theory, however, is developed specifically for the case of parallel, zonal flows. In the Southern Ocean, scale separation between eddies and mean flow is weak at best, and only limited regions of the ACC zonal extent conform to uniform parallel flow. On the other hand, the QG model is predominantly zonal outside of the narrow transition zones. Thus, the QG model may be much more effective in limiting particle exchange through the effects of mean flow advection. This dependence on both mean flow strength and EKE has been discussed previously in the atmospheric literature 
in terms of surf zones and transport barriers (e.g., Juckes and McIntyre 1987). The novelty here is how these processes act in large domains with multiple jets to bring about a reorganization of the flow structure.

\section{b. Transport properties and locality}

In the QG simulations, we introduce topography that consists of ridge and flat bottom sectors, separated by narrow transition regions. A simple hypothesis is that the system adopts, in the appropriate sectors, a flow structure that is equivalent to the flow observed in full ridge or pure flat-bottom simulations with an adjustment between these sectors. This is not the situation observed in these simulations. In particular, for the parameters used in experiment $\mathrm{A}$, the equilibrated flow in a full flat-bottom domain exhibits four, steady, equally spaced jets. Yet, over the flat bottom sector of experiment A we observe five or six jets. Furthermore, in a full ridge simulation, weak transport barriers are supported on the northern flank of the ridge, whereas these northern fronts are only observed near the downstream transition region in experiment $\mathrm{A}$. This behavior supports the hypothesis that local transitions in topography have global implications for flow structure and transport.

To further examine how the ridge influences transport, we employ a mixing diagnostic known as the effective diffusivity introduced by Nakamura (1996) and studied by Shuckburgh and Haynes (2003). The meridional flux of $\mathrm{PV}$ can be described using a diffusive approximation (taking overbars to represent zonal averages),

$$
{\overline{v^{\prime} q^{\prime}}}^{x}=-\kappa_{\mathrm{eff}}(y) \frac{\partial \bar{q}^{x}}{\partial y} \equiv \mathcal{F}
$$

which, in the absence of frictional terms in the upper layer, is independent of $y$ and equal to a constant $\mathcal{F}_{1}$. In (8) $\kappa_{\text {eff. }}(y)$ is the effective diffusivity, which measures tracer contour complexity under the assumption that eddies are responsible for stretching tracer contours. The effective diffusivity is proportional to the ratio of the square of the stirred contour length to the square of the minimum contour length, or equivalently the domain width. Here, the calculation of $\kappa_{\text {eff }}$ is determined from the upper-layer potential vorticity distribution (contours), which in the absence of small-scale dissipation is materially conserved as would be a passive tracer; see further discussion in Thompson (2010). Integration of (8) with respect to $y$ leads to

$$
\mathcal{F}_{1}=\Delta q_{1}\left(\int_{0}^{L_{y}} \frac{1}{\kappa_{\text {eff }}} d y\right)^{-1},
$$

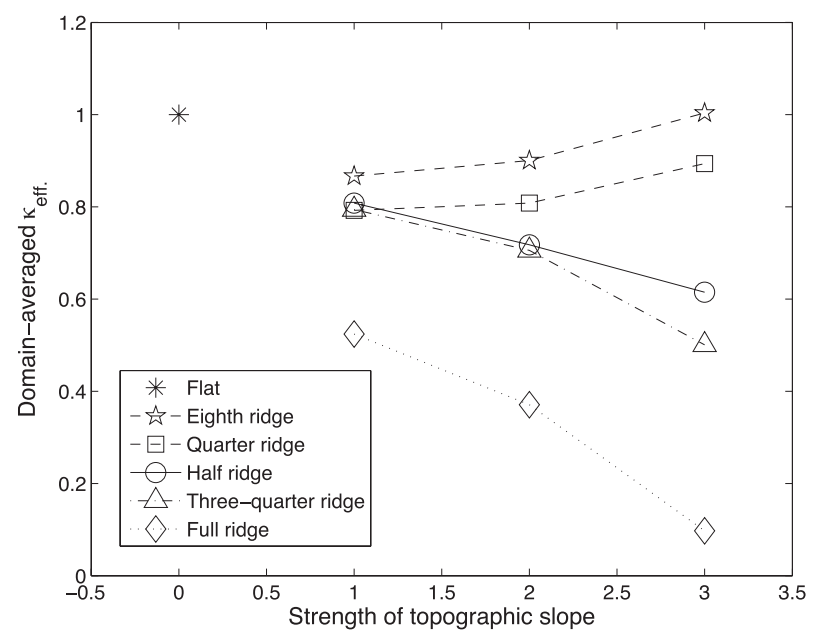

FIG. 11. Ratio of the domain-averaged effective diffusivity [(9)] for varying slope steepness $s$ and ridge extent (symbols) to the simulation with a flat bottom (indicated by the asterisk).

with $\Delta q_{1}=\bar{q}_{1}(0)-\bar{q}_{1}\left(L_{y}\right)$ such that the flux $\mathcal{F}_{1}$ is proportional to the harmonic mean of the effective diffusivity, $1 /\left\langle 1 / \kappa_{\text {eff }}\right\rangle$ (Nakamura 2008), which emphasizes regions of low mixing.

Figure 11 compares the harmonic mean of the effective diffusivity (9), calculated using the upper-layer PV, as a function of ridge height and the extent of the ridge. The results are normalized by the value from a flat bottom simulation. The addition of any topography reduces transport as compared to the flat bottom simulation. When the amplitude of the ridge is weak $(s=1)$, ridge extent has a weak impact on global transport as long as zonal asymmetry exists. As the ridge amplitude increases and the flanks become steeper, the ridge extent leads to different transport properties. A shorter ridge produces greater transport, because the ridge acts more as an island and the variability it generates opposes the stabilizing effect of the ridge's southern flank. For longer ridges, the stabilizing effect of the ridge dominates. Figure 11 confirms that, in general, the global transport cannot simply be determined from the weighted average of transports in full ridge and flat bottom simulations.

Finally, we have briefly explored running longer domain simulations, where the length of the ridge is held fixed and the flat bottom region is extended. By quadrupling the size of the domain we reach a regime where the flow eventually transition back to the four jets observed in the flat bottom simulations. The length scale that characterizes this decay is related to bottom friction as well as eddy-mean flow interactions. Running these longer domain simulations over a range of bottom friction values is beyond the scope of this present study, but 
we are exploring this question of downstream evolution of coherent structures in both idealized and more realistic models.

\section{Summary}

Zonal asymmetries in the dynamics of the ACC are known to occur for many reasons including flow interactions with topography and responses to wind and buoyancy forcing. In this study, two separate approaches suggest that zonal asymmetry acts to localize particle exchange across fronts, as defined by circumpolar contours associated with enhanced property gradients. Using realistic surface velocity fields derived from satellite altimetry and by defining fronts using contours of SSH (Sallée et al. 2008; Sokolov and Rintoul 2007b, 2009), particle exchange is shown to be correlated with EKE in the lee of both large and moderate topography. This echoes the results of Sallée et al. (2011), who show an increase in surface eddy diffusivities within recirculation regions in the lee of topography. However, the analysis used here permits a much finer resolution of the alongstream variations and highlights sharp transitions in the sites of cross-front exchange.

A suite of simulations using a QG model with an idealized zonally asymmetric ridge provides further dynamical insight into front evolution. Again, the combination of strong EKE and a weak mean flow are found to be good indicators of enhanced particle crossing. In the idealized configuration the role of front reorganization as a mechanism for particle exchange is dominant. The change in the structure of the jets typically occurs in localized transition zones and is facilitated by eddy generation through enhanced baroclinicity in the lee of topography. Eddy-mean flow interactions moderate the reorganization of the jets with advection of eddies from generation sites playing an important role. Global properties of the flow, such as the domain-averaged effective diffusivity, may be significantly modified.

A major result of this study is that simple symmetry breaking in a zonally periodic channel can lead to fronts and jets that do not extend throughout the domain. This behavior is particularly relevant to the Southern Ocean where multiple jets are observed to form, split, and merge along the path of the ACC. This suggests that care must be taken in the definition of circumpolar fronts and in the analysis of cross-front fluxes. Transitions in jet structure and the associated transport characteristics are likely to have a strong control over the rate and structure of the Southern Ocean MOC.

Acknowledgments. AFT was supported by a Natural Environment Research Council Advanced Research
Fellowship (NE/H015760/1). We thank two anonymous reviewers, whose comments substantially improved the presentation of the material.

\section{REFERENCES}

Arbic, B. K., and G. R. Flierl, 2004: Effects of mean flow direction on energy, isotropy, and coherence of baroclinically unstable beta-plane geostrophic turbulence. J. Phys. Oceanogr., 34, 77-93.

Belkin, I. M., and A. L. Gordon, 1996: Southern Ocean fronts from the Greenwich meridian to Tasmania. J. Geophys. Res., 101, 3675-3696.

Boland, E. J., A. F. Thompson, E. Shuckburgh, and P. H. Haynes, 2012: The formation of non-zonal jets over sloped topography. J. Phys. Oceanogr., in press.

Böning, C. W., A. Dispert, M. Visbeck, S. R. Rintoul, and F. U. Schwarzkopf, 2008: The response of the Antarctic Circumpolar Current to recent climate change. Nat. Geosci., 1, 864-869.

Bracco, A., and J. Pedlosky, 2003: Local baroclinic instabilities over variable topography in channel flows. J. Phys. Oceanogr., 33, 207-219.

Chelton, D. B., M. G. Schlax, D. L. Witter, and J. G. Richman, 1990: Geosat altimetery observations of the surface circulation of the Southern Ocean. J. Geophys. Res., 95, 17 877-17 903.

,,-- R. M. Samelson, and R. A. de Szoeke, 2007: Global observations of large ocean eddies. Geophys. Res. Lett., 34, L15606, doi:10.1029/2007GL030812.

Drijfhout, S. S., and A. C. Naveira Garabato, 2008: The zonal dimension of the Indian Ocean meridional overturning circulation. J. Phys. Oceanogr., 38, 359-379.

Ferrari, R., and M. Nikurashin, 2010: Suppression of eddy mixing across jets in the Southern Ocean. J. Phys. Oceanogr., 40, 1501-1519.

Fu, L.-L., 2009: Pattern and velocity of propagation of the global ocean eddy variability. J. Geophys. Res., 114, C11017, doi:10.1029/ 2009JC005349.

Gille, S. T., 1997a: Why potential vorticity is not conserved along mean streamlines in a numerical Southern Ocean. J. Phys. Oceanogr., 27, 1286-1299.

_ 1997b: The Southern Ocean momentum balance: Evidence for topographic effects from numerical model output and altimeter data. J. Phys. Oceanogr., 27, 2219-2232.

Haynes, P. H., and E. Shuckburgh, 2000: Effective diffusivity as a diagnostic of atmospheric transport 1: Stratosphere. J. Geophys. Res., 105, 22 777-22 794.

Hogg, A. M., M. P. Meredith, J. R. Blundell, and C. Wilson, 2008: Eddy heat flux in the Southern Ocean: Response to variable wind forcing. J. Climate, 21, 608-620.

Hughes, C. W., and E. R. Ash, 2001: Eddy forcing of the mean flow in the Southern Ocean. J. Geophys. Res., 106, 2713-2722.

Johnson, G. C., and H. L. Bryden, 1989: On the size of the Antarctic Circumpolar Current. Deep-Sea Res., 36, 39-53.

Juckes, M. N., and M. E. McIntyre, 1987: A high-resolution onelayer model of breaking planetary waves in the stratosphere. Nature, 328, 590-596.

Langlais, C., S. R. Rintoul, and A. Schiller, 2011: Variability and mesoscale activity of the Southern Ocean fronts: Identification of a circumpolar coordinate system. Ocean Modell., 39, 79-96.

Lenn, Y. D., T. K. Chereskin, J. Sprintall, and J. L. McClean, 2011: Near-surface eddy heat and momentum fluxes in the Antarctic 
Circumpolar Current in Drake Passage. J. Phys. Oceanogr., 41, 1385-1407.

Le Traon, P. Y., F. Nadal, and N. Ducet, 1998: An improved mapping method of multisatellite altimeter data. J. Atmos. Oceanic Technol., 15, 522-534.

Lu, J., and K. Speer, 2010: Topography, jets and eddy mixing in the Southern Ocean. J. Mar. Res., 68, 479-502.

MacCready, P., and P. B. Rhines, 2001: Meridional transport across a zonal channel: Topographic localization. J. Phys. Oceanogr., 31, 1427-1439.

Marshall, J., and T. Radko, 2003: Residual mean solutions for the Antarctic Circumpolar Current and its associated overturning circulation. J. Phys. Oceanogr., 33, 2341-2354.

— E. Shuckburgh, H. Jones, and C. Hill, 2006: Estimates and implications of surface eddy diffusivity in the Southern Ocean derived from tracer transport. J. Phys. Oceanogr., 36, 1806-1821.

Munk, W. H., and E. Palmèn, 1951: Note on the dynamics of the Antarctic Circumpolar Current. Tellus, 3, 53-55.

Nakamura, N., 1996: Two-dimensional mixing, edge formation, and permiability diagnosed in area coordinates. J. Atmos. Sci., $\mathbf{5 3}, 1524-1537$.

, 2008: Sensitivity of global mixing and fluxes to isolated transport barriers. J. Atmos. Sci., 65, 3800-3818.

Naveira Garabato, A. C., R. Ferrari, and K. Polzin, 2011: Eddy stirring in the Southern Ocean. J. Geophys. Res., 116, C09019, doi:10.1029/2010JC006818.

Nencioli, F., F. d'Ovidio, A. M. Doglioli, and A. A. Petrenko, 2011: Surface coastal circulation patterns by in-situ detection of Lagrangian coherent structures. Geophys. Res. Lett., 38, L17604, doi:10.1029/2011GL048815.

Nikurashin, M., and G. Vallis, 2011: A theory of deep stratification and overturning circulation in the ocean. J. Phys. Oceanogr., 41, 485-502.

Olbers, D., and M. Visbeck, 2005: A model of the zonally averaged stratification and overturning in the Southern Ocean. J. Phys. Oceanogr., 35, 1190-1205.

Orsi, A. H., T. W. Whitworth III, and W. D. Nowlin, 1995: On the meridional extent and fronts of the Antarctic Circumpolar Current. Deep-Sea Res., 42, 641-673.

Pavan, V., and I. Held, 1996: The diffusive approximation for eddy fluxes in baroclinically unstable jets. J. Atmos. Sci., 53, 1262-1272.

Pedlosky, J., 1987: Geophysical Fluid Dynamics. 2nd ed. SpringerVerlag, $710 \mathrm{pp}$.

Radko, T., and J. Marshall, 2006: The Antarctic Circumpolar Current in three dimensions. J. Phys. Oceanogr., 36, 651-669.

Rio, M.-H., P. Schaeffer, F. Hernandez, and J. M. Lemoine, 2005: The estimation of the ocean mean dynamic topography through the combination of altimetric data, in situ measurements and GRACE geoid: From global to regional studies. Proc. GOCINA Int. Workshop, Luxembourg, Centre Européen de Géodynamique et de Séismologie.

Sallée, J. B., K. Speer, and R. Morrow, 2008: Southern Ocean fronts and their variability to climate modes. J. Climate, 21, 3020-3039.
, and S. R. Rintoul, 2010: Zonally asymmetric response of the Southern Ocean mixed-layer depth to the southern annular mode. Nat. Geosci., 3, 273-279.

,$- \ldots$, and -2011 : Mean-flow and topography control on surface eddy-mixing in the Southern Ocean. J. Mar. Res., 69, 753-777.

Shuckburgh, E., and P. H. Haynes, 2003: Diagnosing transport and mixing using a tracer-based coordinate system. Phys. Fluids, 15, 3342-3357.

Smith, K. S., 2007: Eddy amplitudes in baroclinic turbulence driven by nonzonal mean flow: Shear dispersion of potential vorticity. J. Phys. Oceanogr., 37, 1037-1050.

- , G. Boccaletti, C. C. Henning, I. N. Marinov, C. Y. Tam, I. M. Held, and G. K. Vallis, 2002: Turbulent diffusion in the geostrophic inverse cascade. J. Fluid Mech., 469, 13-48.

Sokolov, S., and S. R. Rintoul, 2007a: On the relationship between fronts of the Antarctic Circumpolar Current and surface chlorophyll concentrations in the Southern Ocean. J. Geophys. Res., 112, C07030, doi:10.1029/2006JC004072.

$\longrightarrow$, and - 2007b: Multiple jets of the Antarctic Circumpolar Current south of Australia. J. Phys. Oceanogr., 37, 13941412.

$\longrightarrow$, and $—$, 2009: Circumpolar structure and distribution of the Antarctic Circumpolar Current fronts: 1. Mean circumpolar paths. J. Geophys. Res., 114, C11018, doi:10.1029/2008JC005108.

Thompson, A. F., 2010: Jet formation and evolution in baroclinic turbulence with simple topography. J. Phys. Oceanogr., 40, 257-278.

—, and W. R. Young, 2007: Two-layer baroclinic eddy heat fluxes: Zonal flows and energy balance. J. Atmos. Sci., 64, 3214-3231.

_ , and K. J. Richards, 2011: Low frequency variability of Southern Ocean jets. J. Geophys. Res., 116, C09022, doi:10.1029/ 2010JC006749.

— , P. H. Haynes, C. Wilson, and K. J. Richards, 2010: Rapid Southern Ocean front transitions in an eddy-resolving ocean GCM. Geophys. Res. Lett., 37, L23602, doi:10.1029/2010GL045386.

Toggweiler, J. R., and B. Samuels, 1995: Effect of Drake Passage on the global thermohaline circulation. Deep-Sea Res. II, 42, 477-500.

Treguier, A. M., M. H. England, S. R. Rintoul, G. Madec, J. Le Sommer, and J.-M. Molines, 2007: Southern Ocean overturning across streamlines in an eddying simulation of the Antarctic Circumpolar Current. Ocean Sci., 3, 491-507.

Visbeck, M., J. Marshall, T. Haine, and M. Spall, 1997: Specification of eddy transfer coefficients in coarse-resolution ocean circulation models. J. Phys. Oceanogr., 27, 381-402.

Williams, R. G., C. Wilson, and C. W. Hughes, 2007: Ocean and atmosphere storm tracks: The role of eddy vorticity forcing. J. Phys. Oceanogr., 37, 2267-2289.

Witter, D. L., and D. B. Chelton, 1998: Eddy-mean flow interaction in zonal oceanic jet flow along zonal ridge topography. J. Phys. Oceanogr., 28, 2019-2039. 